\title{
Discrimination of Normal and Abnormal Heart Sounds Using Probability Assessment
}

\author{
Filip Plesinger, Juraj Jurco, Pavel Jurak, Josef Halamek \\ ISI of the CAS, Brno, Czech Republic
}

\begin{abstract}
Aims: According to the "2016 Physionet/CinC Challenge", we propose an automated method identifying normal or abnormal phonocardiogram recordings.

Method: Invalid data segments are detected (saturation, blank and noise tests). The record is transformed into amplitude envelopes in five frequency bands. Systole duration and RR estimations are computed; $15-90 \mathrm{~Hz}$ amplitude envelope and systole/RR estimations are used for detection of the first and second heart sound (S1 and S2). Features from accumulated areas surrounding $S 1$ and $S 2$ as well as features from the whole recordings were extracted and used for training. During the training process, we collected probability and weight values of each feature in multiple ranges. For feature selection and optimization tasks, we developed C\# application PROBAfind, able to generate the resultant Matlab code.

Results: The method was trained with 3153 Physionet Challenge recordings (length 8-60 seconds; 6 databases). The results of the training set show the sensitivity, specificity and score of 0.93, 0.97 and 0.95, respectively. The method was evaluated on a hidden Challenge dataset with sensitivity and specificity of 0.77 and 0.91, respectively. These results led to an overall score of 0.84 .
\end{abstract}

\section{Introduction}

This paper describes a method for automated discrimination of normal and abnormal heart sounds, according to Physionet Challenge 2016 [1]. The presented method introduces a probability assessment from multiple features. The used features are mostly derived from the accumulated systole and diastole area. Moreover, some features are independent of heart sounds detection.

\section{Method}

The workflow of the method is presented in Fig. 1. It consists of the generation of amplitude envelopes in 5 frequency bands, detection of invalid areas, detection of heart sounds S1 and S2, extracting features, limit test and computing and comparing the resultant probability.

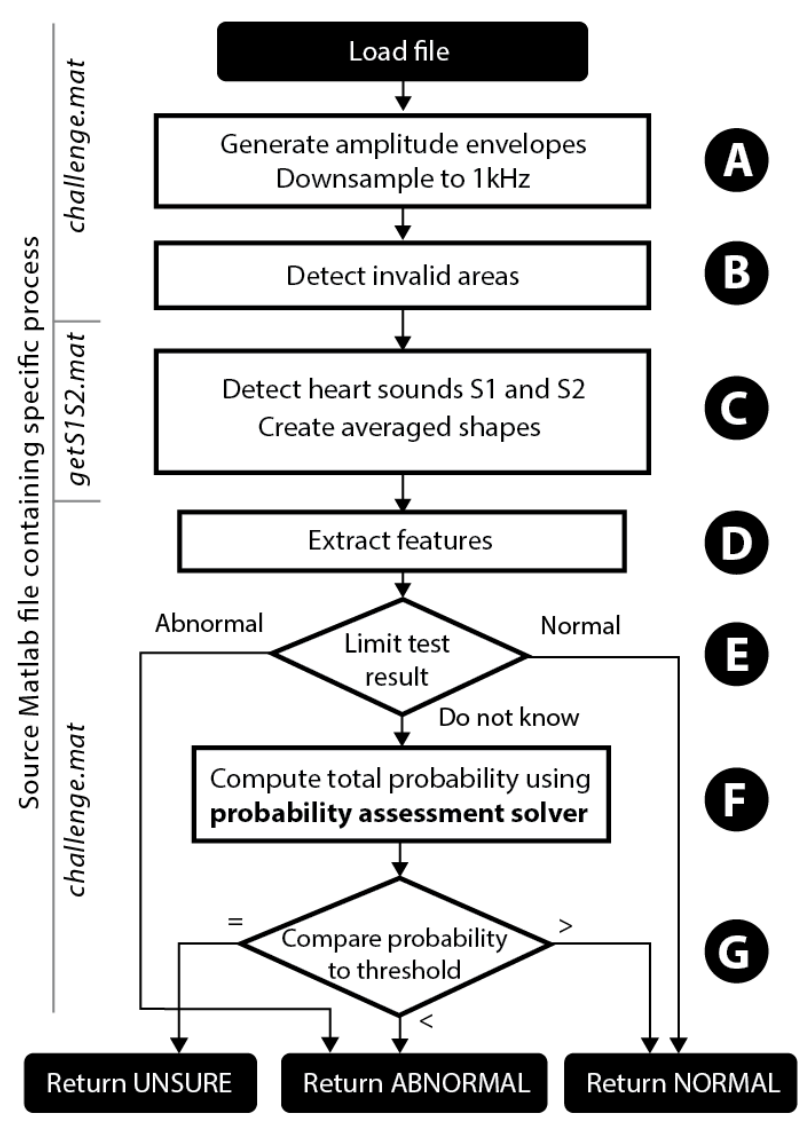

Figure 1. Flowchart of presented method

\subsection{Pre-processing}

Five amplitude envelopes are generated from the input wave file (sampling at $2 \mathrm{kHz}$ ) using Hilbert and Fourier transformations. The generated envelopes use the bands $15-90 \mathrm{~Hz}$ (LF), $55-150 \mathrm{~Hz}$ (MF), 100-250 Hz (HF), 200$450 \mathrm{~Hz}$ (SF) and 400-800 Hz (UF). All generated envelopes and source signal are down-sampled to $1 \mathrm{kHz}$ (Fig. 1A). The LF envelope is used in $\mathrm{S} 1$ and $\mathrm{S} 2$ 
detection; the EF and LF ratio is used for invalid area detection. The LF, MF, HF, UF and SF envelopes (Fig. 2) are used in the limits check and probability assessment solver.

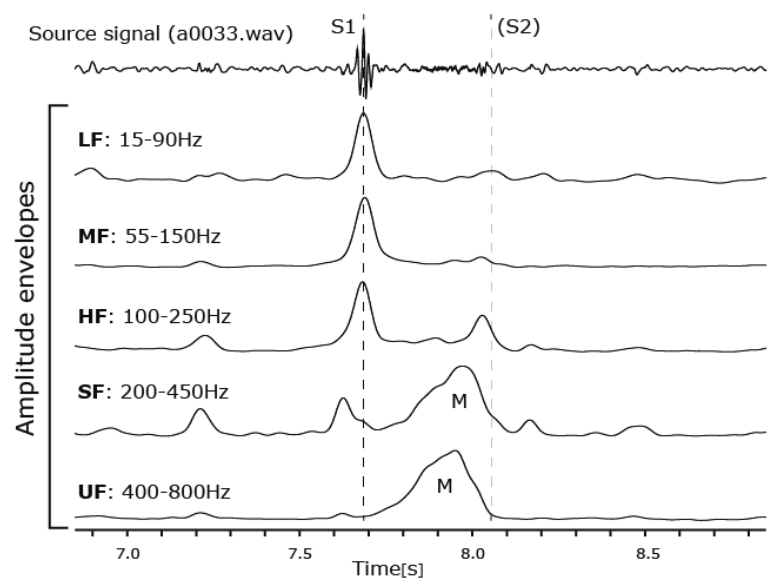

Figure 2. Abnormal heart sounds (record a0033.wav) and computed amplitude envelopes (smoothed with LP filter). $\mathrm{S} 1$ and S2 are the first and the second heart sound, respectively. $M$ is audible murmur, visible in the two highest frequency bands SF and UF. The image was rendered using SignalPlant software [2].

\subsection{Detection of invalid areas}

Invalid areas are detected in a $1 \mathrm{~s}$ moving window with a $0.5 \mathrm{~s}$ step (Fig. 1B). A histogram (10 bins) from the raw signal is made. If the histogram leads to a non-usual signal distribution (based on [3]) or the sum of the HF envelope is higher than the sum of the LF envelope, the block is marked as "invalid".

\subsection{S1 and S2 heart sound detection}

Prior to the heart sound detection, an estimation of the systole duration and RR interval is made. Systole duration estimation $S_{D E}$ (Fig. 3A) uses the LF amplitude envelope. Peaks are detected (threshold $74^{\text {th }}$ percentile, minimal peak distance $175 \mathrm{~ms}$ ) and a histogram of their distances is built. Due to the low variability of the systole duration, the most common bin in the histogram should lead to systole duration. RR estimation $\left(R R_{E}\right)$ is based on autocorrelation of the LF envelope (Fig. 3B). In the resultant autocorrelation function peaks are found (threshold $80^{\text {th }}$ percentile, min. peak distance $300 \mathrm{~ms}$ ). Peak differences are collected (excluding nonphysiological RR distances) and the RR interval is computed as a median of the peak distances. In case of fewer than 3 distances, the estimated RR interval is computed as $2.8 \times S_{D E}$. The $\mathrm{RR}$ to systole ratio is compared (Fig. 3C) to the limits $(>2.1 ;<4.5)$. If it does not pass, the $S_{D E}$ or $R R_{E}$ is corrected. The heart sound extraction block (Fig. 3D) searches in peaks derived from the LF envelope and finds S1 and S2 pair locations. If the number of resultant pairs is lower than 2, the process is aborted (Fig. 3E).

\section{Amplitude envelopes, invalid data locations}

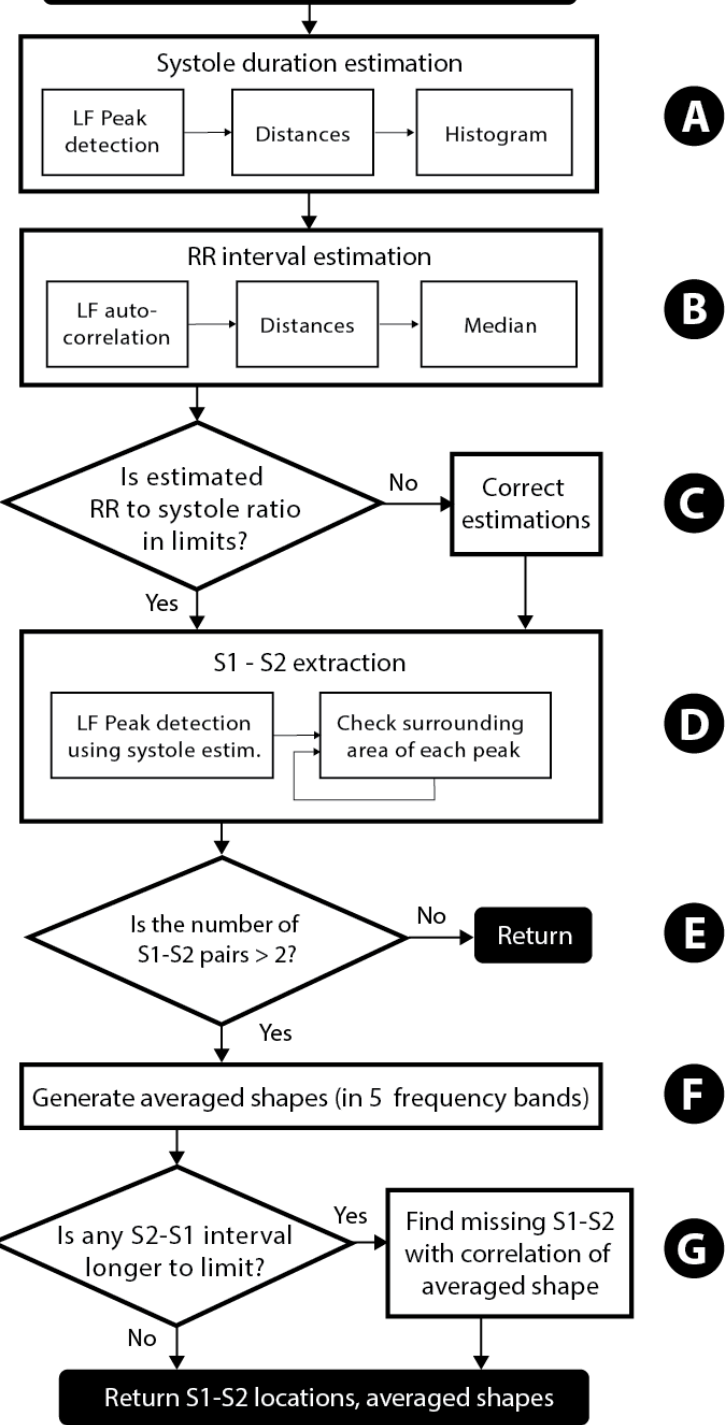

Figure 3. Flowchart of proposed heart sound detector.

Averaged shapes of systole and diastole area are generated in each of the used frequency bands (Fig. 3F).

These averaged shapes are intended for feature collection as well as for missing S1/S2 detection check. If any $\mathrm{S} 2-\mathrm{S} 1$ interval (i.e. diastole) is longer than $1.8 \mathrm{~s}$ (Fig. $3 \mathrm{G})$, the LF average shape of the $\mathrm{S} 1-\mathrm{S} 2$ area is used to find missing heart sounds with correlation (threshold 0.8). 


\subsection{Investigated features}

We extracted 215 features for further investigation. These features were statistical properties of segments of averaged heart sound envelopes in several time segments (before $\mathrm{S} 1$, at $\mathrm{S} 1$, after $\mathrm{S} 1$, before $\mathrm{S} 2$, at $\mathrm{S} 2$, after $\mathrm{S} 2$ and diastole) in five frequency ranges. The ratios between those properties were also used.

Moreover, we generated features independent of detection of S1 and S2. We tested the skewness and kurtosis of all the generated envelopes. We also designed a transformation reacting to continuous blocks of a similar sound:

$$
C S_{i}=\log \left(\operatorname{Corr}\left(F F T_{i}, F F T_{i-s t e p}\right) \times \sum F F T_{i}\right)
$$

where $F F T_{i}$ is the absolute value of the Fast Fourier transform (window $128 \mathrm{~ms}$ ) following a sample with index $i$. This transformation is able to reveal murmurs, as shown in Fig. 4.
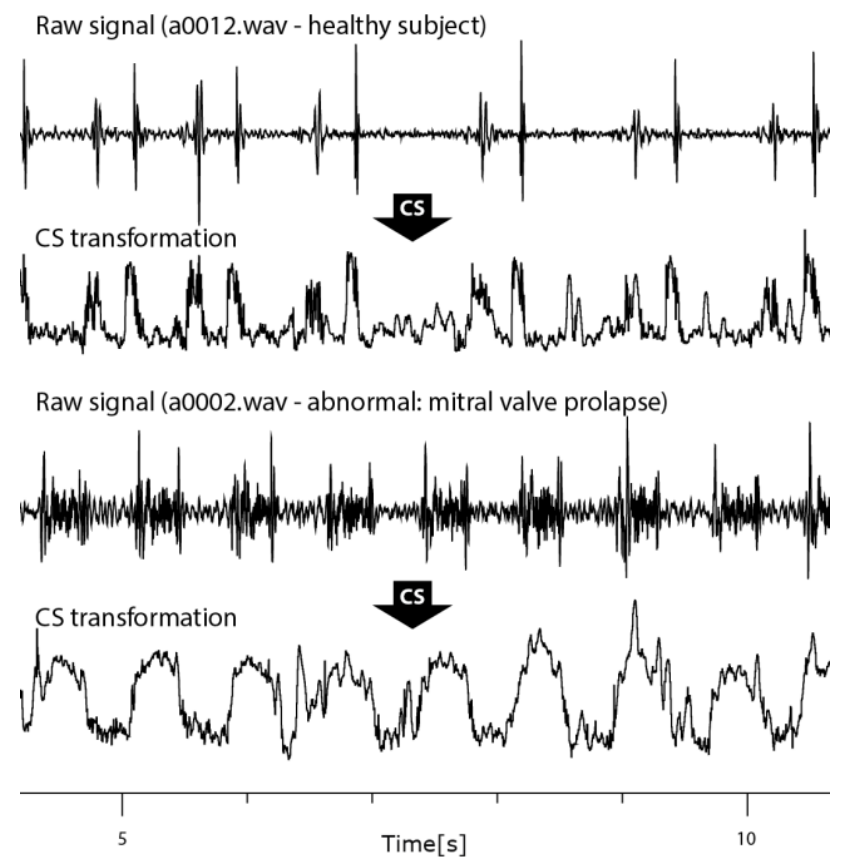

Figure 4. CS transformation of a signals containing normal and abnormal heart sounds. It positively reacts to continuous sounds of a similar frequency image, revealing murmurs.

\subsection{Limit test - preliminary classification}

Some of the extracted features were used for the raw classification of normal (abnormal) sounds (Fig. 1E). Feature thresholds were set to separate only normal (resp. abnormal) records from the rest. If this limit test points to normality (abnormality), further processing is skipped.
Table 1 shows the number of futures used for the limit test and its ability to reveal normal and abnormal sounds for each tested dataset.

Table 1. Count of features used for the preliminary classification of normal $\left(\mathrm{N}_{\mathrm{PN}}\right)$ and abnormal $\left(\mathrm{N}_{\mathrm{PA}}\right)$ sounds in each dataset. No false negative detection was allowed during training. $\mathrm{N}(\mathrm{A})$ is the relative number of normal (abnormal) sounds in the dataset. All (1) features were independent on heart sounds detection, while All (2) used them. Datasets $\mathrm{A}$ and $\mathrm{F}$ were excluded due to their absence in test set; $\mathrm{C}$ was excluded because it can be divided using only one parameter.

\begin{tabular}{lllll}
\hline Dataset & $\mathrm{N}_{\mathrm{PN}}$ & $\%$ of $\mathrm{N}$ & $\mathrm{N}_{\mathrm{PA}}$ & $\%$ of A \\
\hline All (1) & 2 & $40 \%$ & 1 & $17 \%$ \\
All (2) & 4 & $33 \%$ & 7 & $16 \%$ \\
B & 4 & $16 \%$ & 3 & $16 \%$ \\
D & 2 & $54 \%$ & 4 & $25 \%$ \\
E & 3 & $96 \%$ & 5 & $66 \%$ \\
\hline
\end{tabular}

\subsection{Training - probability assessment solver}

Because no single feature (except dataset $\mathrm{C}$ ) could be used for a reliable division into normal and abnormal sounds, multiple features were used for each dataset. Multiple features usually lead to machine learning methods such as neural networks [4] or supported vector machines [5]. Contrary to these standard approaches, we decided to design a new method using the probability of a normal/abnormal sound in single sections in each feature.

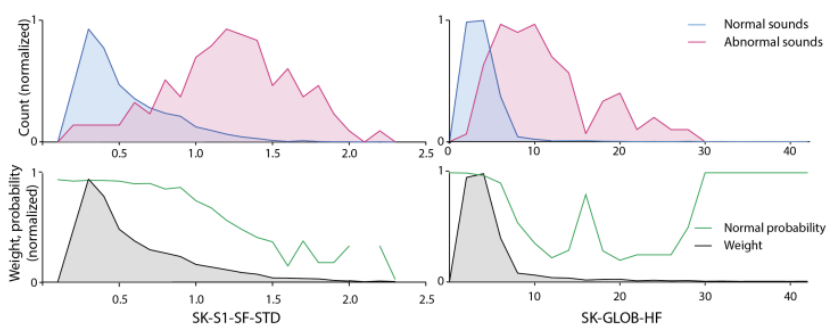

Figure 5. Example of two features (dataset E). Left standard deviation of skewness of averaged S1 surroundings in the SF frequency band, right - skewness of the record HF amplitude envelope. Blue - occurrence of normal sounds, red - occurrence of abnormal sounds, black - weight, green - normal sound probability.

For each feature, two histograms (20 bins) were built for the normal/abnormal values (Fig. 5). The weight of each histogram bin was computed as the ratio between normal and abnormal occurrences in the bin to the total number of subjects. The resultant probability is transformed with the sigmoid function and added to the probability from the other parameters (Fig. 1F). The summed probability is compared to the manually selected 
threshold (Fig. 1G) leading to a final statement about heart-sounds normality. For the inspection and automatized feature selection process, we built PROBAfind software (http://medisig.com/probafind). It also allows interactive setting of the sensitivity/specificity ratio and generates a Matlab code for challenge entry.

\subsection{Probability assessment - selected features}

Due to the different behaviour of the same features in different datasets (containing same pathology, as in Fig. 6) we decided to build separate feature set for each dataset. For test-only datasets $\mathrm{G}$ and I, general feature set was built. Features were selected semi-automatically using PROBAfind software. Table 2 shows the number of used features and the strongest feature for the training datasets. Dataset $\mathrm{C}$ (containing mitral regurgitation and mitral stenosis) was not subjected to the probability assessment; it can be divided using just one parameter.

Table 2. Selected features for training datasets. $\mathrm{N}$ shows the total number of selected features for each dataset. "Other" is general dataset in case that the dataset is not known or cannot be identified. Dataset $\mathrm{C}$ does not use probability assessment, it uses simple comparison instead.

\begin{tabular}{|c|c|c|}
\hline Set & $\mathrm{N}$ & The strongest feature \\
\hline A & 13 & Diastole value in UF to LF ratio (median) \\
\hline B & 11 & $\begin{array}{l}\text { Ratio between value in S1 to after-S1 area } \\
\text { in accumulated LF (median) }\end{array}$ \\
\hline $\mathrm{C}^{*}$ & 1 & Skewness of accumulated $\mathrm{S} 2$ in $\mathrm{HF}$ \\
\hline $\mathrm{D}$ & 2 & $\begin{array}{l}\text { Ratio between value in } \mathrm{S} 1 \text { to after } \mathrm{S} 1 \text { area } \\
\text { in accumulated LF }\end{array}$ \\
\hline $\mathrm{E}$ & 4 & Skewness of accumulated S1 in UF \\
\hline $\mathrm{F}$ & 7 & Mean of accumulated area after $\mathrm{S} 2$ in LF \\
\hline Other & 13 & Skewness of accumulated S1 in UF \\
\hline
\end{tabular}
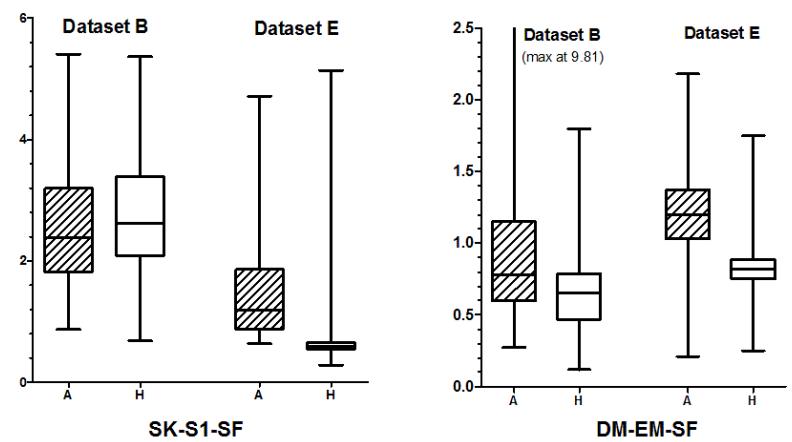

Figure 6. Feature heterogeneity in datasets $\mathrm{B}$ and $\mathrm{E}$ with coronary artery disease (CDA). Presented features (SKS1-SF - skewness of accumulated S1 area in SF band; DM-EM-SF - ratio between S2 and the preceding area in the SF band) separate healthy subjects $(\mathrm{H})$ from CDA patients (A) in dataset $\mathrm{E}$. The same parameters are less usable in dataset $\mathrm{B}$.

\section{Results and conclusion}

The training and testing results are presented in Table 3. Testing was carried out on a hidden test set. The presented method consumed $5.11 \%$ (average per record) of instructions allowed.

Table 3. Training and testing results. $\mathrm{N}-$ number of records, $\mathrm{Se}$ - sensitivity, $\mathrm{Sp}$ - specificity.

\begin{tabular}{lllll}
\hline Dataset & N & Se & Sp & Score \\
\hline Training set & 3153 & 0.93 & 0.97 & 0.95 \\
Test set (hidden) & 500 & 0.77 & 0.91 & 0.84 \\
\hline
\end{tabular}

An approach for the automated detection of normal/abnormal heart sounds using a probability assessment was introduced. We also proposed features independent of heart sound detection, which may be suitable for hardware implementation.

\section{Acknowledgements}

Research was supported by project no. P102/12/2034 from the Grant Agency of the Czech Republic and by MEYS CR (LO1212), its infrastructure by MEYS CR and EC (CZ.1.05/2.1.00/01.0017) and by ASCR (RVO:68081731).

\section{References}

[1] C. Liu, D. Springer, Q. Li, B. Moody, R. A. Juan, F. J. Chorro, F. Castells, J. M. Roig, I. Silva, A. E. W. Johnson, Z. Syed, S. E. Schmidt, C. D. Papadaniil, L. Hadjileontiadis, H. Naseri, A. Moukadem, A. Dieterlen, C. Brandt, H. Tang, M. Samieinasab, M. R. Samieinasab, R. Sameni, R. G. Mark, and G. D. Clifford, "An open access database for the evaluation of heart sound algorithms," Physiol. Meas., vol. 37, no. 9, 2016.

[2] F. Plesinger, J. Jurco, J. Halamek, and P. Jurak, "SignalPlant: an open signal processing software platform," Physiol. Meas., vol. 37, no. 7, pp. N38-N48, 2016.

[3] F. Plesinger, P. Klimes, J. Halamek, and P. Jurak, "Taming of the monitors: reducing false alarms in intensive care units," Physiol. Meas., vol. 37, no. 8, p. 1313, 2016.

[4] J. P. De Vos and M. M. Blanckenberg, "Automated pediatric cardiac auscultation," IEEE Trans. Biomed. Eng., vol. 54, no. 2, pp. 244-252, 2007.

[5] I. Maglogiannis, E. Loukis, E. Zafiropoulos, and A. Stasis, "Support Vectors Machine-based identification of heart valve diseases using heart sounds," Comput. Methods Programs Biomed., vol. 95, no. 1, pp. 47-61, 2009.

Address for correspondence:

Filip Plesinger, ISI of the CAS

Kralovopolska 147 ,

Brno 612 64, Czech Republic

E-mail address: fplesinger@isibrno.cz 